\title{
APLICATIVO PARA VALIDAR A EFETIVIDADE DE OBJETOS DE APRENDIZAGEM: UTILIZANDO OS MÉTODOS MMEEBB E MCE
}

PATOS DE MINAS/MG MAIO/2018

\author{
Mislene Dalila da Silva - UFU - mislenedalila@gmail.com \\ Gleicy Tatiane Lima - UNIPAM - gleicylima@unipam.edu.br \\ Luciano Vieira Lima - UFU - avaliacoesefetivas@gmail.com \\ Mirian Camila da Silva - UFU - miriancs@unipam.edu.br \\ Sara Luzia Melo - UFU - saraluziamelo@gmail.com \\ Adriene Sttéfane Silva - UFU/ UNIPAM - mis.lila@hotmail.com
}

Tipo: Relato de Experiência Inovadora (EI)

Categoria: Métodos e Tecnologias

Setor Educacional: EDUCAÇÃO MÉDIA E TECNOLÓGICA, EDUCAÇÃO SUPERIOR,
EDUCAÇÃO CORPORATIVA, EDUCAÇÃO CONTINUADA EM GERAL

\section{RESUMO}

A presente pesquisa buscou validar a efetividade dos objetos de aprendizagem que são disponibilizados para os alunos atualmente através de um aplicativo. Um objeto de aprendizagem deve ser chamado dessa forma se de fato efetivar a aprendizagem. Portanto o estudo foi desenvolvido em um ambiente onde $o$ aluno tem acesso a objetos de aprendizagem que serão avaliados quanto à fixação do conhecimento na memória do indivíduo utilizando o Método de Memorização Exponencial Efetivo na Base Binária - MMEEBB, e em paralelo trabalhou-se também o Mapa de Conhecimento Estruturado - MCE onde o aluno não passa para um novo conhecimento sem que o anterior esteja preenchido. Assim se o aluno tiver uma dificuldade para seguir para o próximo conteúdo ele contará com o apoio do Tutor/Professor, que receberá um relatório com informações sobre a dificuldade de cada aluno, podendo atuar de forma personalizada na dificuldade. Dessa forma um objeto de avaliação é inserido a cada objeto de aprendizagem ministrado, para averiguar se realmente a aprendizagem foi consolidada. Seguindo os métodos do mapa de conhecimento estruturado, se o aluno não conseguir comprovar por meio da avalição ou uma prática/ação, inserida pelo tutor/professor o mesmo não pode passar para o próximo conteúdo, sendo necessário receber mais conteúdos e ter o apoio dos tutores/professores para sanar suas dúvidas. Sendo assim esse aplicativo desenvolvido que avalia a efetividade de objetos de aprendizagem, permite quantificar e qualificar a aprendizagem do aluno. Possibilitando a geração de relatórios e registros que identifica de maneira personalizada a dificuldade que cada um possui. Possibilitando uma melhor atuação da instituição quanto à dificuldade de cada estudante. Para alcançar o objetivo deste trabalho foi realizada uma pesquisa exploratória e descritiva sobre resultados do uso de objetos de aprendizagem no ambiente de ensino e aprendizagem.

Palavras-chave: Objetos de Aprendizagem; Efetividade da Aprendizagem; Mapa de Conhecimento Estruturado; Método de Memorização Exponencial Efetivo na Base Binária;

Educação a Distância 


\section{INTRODUÇÃO}

"O mundo não é mais o mesmo", frase típica das gerações anteriores. Eles não estão errados, pois a nova geração é ansiosa, impaciente e tem mudado todos os formatos congelados e obsoletos no qual a sociedade estava acostumada. Isso tem feito com que tudo acabe se transformando e na maioria das vezes para melhor. Desde pedir uma pizza até a forma de pagar uma conta mudou, ficando mais prático e interativo. A interatividade tomou grandes proporções nos dias atuais e a internet proporcionou uma grande mudança no paradigma pedagógico, e até mesmo na maneira em que as pessoas aprendem e ensinam.

A educação demanda novos formatos de ensino nas escolas, nos materiais, nos métodos e os discentes buscam por esses recursos tecnológicos a fim de um melhor aprendizado, que se adapte a sua vida cotidiana. Isso requer a necessidade de novos conhecimentos, buscando constantemente qualificação, orientação e processos de interação com novas tecnologias. Durante o processo de ensino-aprendizagem, de acordo com a ideia da aprendizagem significativa, o aprendiz necessita ter uma experiência individual e pessoal ao consultar o material didático utilizado na abordagem de determinado conteúdo. Com base nesse requisito, busca-se no uso da interatividade a solução para o desenvolvimento cognitivo mais eficiente do aprendiz. (TAVARES, 2003.)

Partindo desta visão, o uso de ferramentas que construam e avaliem o ensino individualmente garantem que o educador possa tratar de forma mais assertiva e personalizada no ensino. Baseando-se nessas mudanças, cada vez mais instituições de ensino têm investido todos seus esforços em recursos da nova tecnologia para complementar e melhorar o método ensino-aprendizagem. Um desses recursos chamase objetos de aprendizagem, que seriam basicamente recursos importantes usados para compor o processo de ensino-aprendizagem. Mas nem sempre esses objetos de aprendizagem são efetivos, normalmente replicam o conteúdo de um PDF e principalmente com o tempo o mesmo torna-se obsoleto. Principalmente utilizando a lógica de um game, quando se "conclui" um jogo a motivação para jogá-lo novamente se atenua, pois o desafio foi concluído.

O Objetivo deste trabalho foi desenvolver um aplicativo que efetiva os Objetos de Aprendizagem - OAs na educação, através de objetos avaliativos após a utilização dos mesmos. O estudo foi desenvolvido em um ambiente onde o aluno terá acesso a objetos de aprendizagem que serão avaliados quanto à fixação do conhecimento na memória do indivíduo, e em paralelo trabalhar o mapa de conhecimento estruturado onde o aluno 
não passa para um novo conhecimento sem que o anterior esteja preenchido. Para alcançar o objetivo deste trabalho foi realizada uma pesquisa exploratória e descritiva sobre resultados do uso de objetos de aprendizagem no ambiente de ensino e aprendizagem.

Desta forma, a proposta desta pesquisa é que os objetos de aprendizagem sejam efetivos e de fato façam o devido papel que é a consolidação da aprendizagem. Portanto o desenvolvimento do aplicativo foi balizado por métodos de reforço e efetivação da aprendizagem como o Mapa de Conhecimento Estruturado - MCE e o Método de Memorização Exponencial Efetivo na Base Binária - MMEEBB.

\section{REFERENCIAL TEÓRICO}

\subsection{CONSOLIDANDO A APRENDIZAGEM}

Depois de muitas pesquisas Miller (1956), que estuda a memória, conclui que a capacidade de informações que não envolvem processos cognitivos de um indivíduo é 7+-2, ou seja, o ser humano retém na memória no mínimo cinco e no máximo nove informações. Quando a informação é muito complexa, a mente humana divide em blocos, dessa forma fica mais simples a memorização de um número grande ou informações desordenadas.

Nos estudos de Cowan (2001), relata-se que na memória de curto prazo, é possível reter apenas quatro informações ao mesmo tempo, e ainda afirma que para cada nova informação é necessário que se abra mão de outra mantida no cérebro até então. Segundo Sweller (2003), se aprende melhor quando a quantidade de informações passadas ao aluno for conciliável com a capacidade de compreensão humana.

O Método de Memorização Exponencial Efetivo na Base Binária - MMEEBB, consegue adaptar e resolver essas problemática de memória. O método é o inverso conceitual da Forgeting Curve de Hermann Ebbinghaus. Isso porque o MMEEBB apresenta a Curva de Memorização Efetiva - CME gerada pelo Intervalo do Reforço do Aprendizado - IRA em dias e o Hermann Ebbinghaus propôs a curva de esquecimento. (FERREIRA et al, 2014).

A CME determina quando o indivíduo tem que recordar a informação para reter o conhecimento em sua memória. A tendência do conhecimento é manter perene de acordo com o reforço $2^{n}$. A cada $2^{n}$ o conhecimento recebido deve ser relembrado para que a informação seja consolidada. Isso através da utilização do processo exponencial 
de potência na base 2 para se obter o valor, em dias, do intervalo de reforço do aprendizado, que mantém o conhecimento permanentemente disponível na memória de longo prazo, descartando-se problemas de patologias cerebrais no aprendiz.

Fato testado empiricamente por Dias et al (2009), no qual, depois de certo tempo, o $2^{n}$, apresenta valores em décadas. Isto demonstra o fato de pessoas idosas recordarem todos os fatos de sua infância e adolescência e não recordar fatos da semana anterior, por exemplo. Este fenômeno é explicado devido ao fato de a memória guardar e ter acessos às informações através de pontes eletroquímicas.

\subsection{MAPA DE CONHECIMENTO ESTRUTURADO}

O cérebro é o "computador central" do nosso corpo e uma das ferramentas mais avançadas que temos, porém ele não é multitarefa em processos cognitivos, por esse motivo é importante que o conhecimento seja passado de forma ordenada ao aluno, evitando que ele passe para um novo conhecimento sem ter abstraído da melhor forma o conteúdo anterior. O Mapa de Conhecimento Estruturado - MCE identifica de forma personalizada o conteúdo a ser passado para o aluno, não deixando o mesmo passar para um novo conhecimento sem que o anterior esteja fixado em sua memória.

Partindo desta visão, o uso das técnicas de memorização em intervalos de tempo préestabelecidos pela taxa IRA do MMEEBB é garantir que o indivíduo não perca 0 conhecimento adquirido, bem como facilitar á a retenção dos conhecimentos anteriores na aquisição de um novo.

\subsection{OBJETOS DE APRENDIZAGEM}

Quando se refere a objetos de aprendizagem associa-se ao uso do computador e da internet. (MIRANDA 2004, p.14). Segundo Sosteric; Hesemeier (2002), os objetos de aprendizagem são todos recursos digitais utilizados no ensino-aprendizagem, como, por exemplo, filmes ou imagens. Para os pesquisadores do Learning Object Metadata (LOM) do Institute of Electrical and Electronics Engineers (IEEE) objetos de aprendizagem é qualquer entidade, digital ou não digital, que pode ser usada, reutilizada ou referenciada durante a aprendizagem apoiada pelo uso de tecnologia (IEEE, 2002, p.6).

$\mathrm{Na}$ educação à distância a utilização de OAs, necessita da inserção de metadados que permitam ser armazenados e referenciados em uma base de dados global. Para que sejam reutilizados em diferentes contextos. Outro ponto a ser avaliado é a usabilidade a acessibilidade do mesmo tratando principalmente a interoperabilidade, quanto a 
utilização do mesmo a partir de diversas plataformas e dispositivos. Assim a definição de OA está referenciada em uma unidade de conteúdo digital, tendo como principal objetivo a habilidade de reuso em diferentes contextos educacionais. (NIKOLOPOULOS et al., 2012, p. 113).

Existem diversos conceitos citados por vários autores do que é um objeto de aprendizagem, e mesmo assim, eles não divergem muito em suas opiniões. Para a autora Behar (2009), objetos de aprendizagem são "qualquer material digital, por exemplo, texto, animações, vídeos, imagens, aplicações, páginas web de forma isolada ou em combinação com fins educacionais".

Portanto um objeto virtual de aprendizagem é um recurso digital reutilizável que auxilie na aprendizagem de algum conceito e, ao mesmo tempo estimule o desenvolvimento de capacidades pessoais, como, por exemplo, imaginação e criatividade. Dessa forma, um objeto virtual de aprendizagem pode tanto contemplar um único conceito quanto englobar todo o corpo de uma teoria. Pode ainda compor um percurso didático, envolvendo um conjunto de atividades, focalizando apenas determinado aspecto do conteúdo envolvido, ou formando, com exclusividade, a metodologia adotada para determinado trabalho. (SPINELLI, 2007, p.7)

\section{RESULTADOS}

Quando se pode garantir que o uso de objetos de aprendizagem teve o sucesso esperado? Para que se possa avaliar a efetividade do uso de objetos de aprendizagem, eles precisam inicialmente ter suas características bem definidas, verificando se a qualidade do conteúdo está devidamente balizada e resumida com informações precisas, se o OA é claro, flexível, reutilizável e relevante. A facilidade de uso do mesmo também deve ser questionada, avaliando se ele é engajador, se suas instruções são claras, e o quão interativo e visualmente atraente ele é.

O objetivo do uso de OA deve ser o ensino e o aprendizado, priorizando o aprendiz. Combinar recursos de OA com o modelo pedagógico deve possibilitar que o professor supervisione a execução das práticas didáticas de seus alunos e a partir delas coletar dados a fim de verificar a efetividade dos sistemas e corrigir o que for necessário. A simples construção de um ambiente não indica o caminho do sucesso em uma perspectiva educacional. (MOREIRA, et al. 2006). Por esse motivo, o modelo pedagógico personalizado com objetos de aprendizagem deve ter todos seus resultados estudados e parametrizados para balizar a efetividade do uso dos mesmos. 
Portanto para testar se de fato os objetos de aprendizagem são efetivos, foi desenvolvido um aplicativo para avaliar o desempenho do aluno após cada objeto de aprendizagem visualizado, balizados pelos mapas de conhecimento estruturado e método de memorização exponencial efetivo na base binária. A seguir se apresenta através de prints a interface do sistema e seu funcionamento.

O aplicativo pode ser utilizado na modalidade de ensino presencial e a distância. Seu funcionamento inicia-se após o acesso, na visão do aluno através da seleção de uma disciplina. Como pode se observar na Figura 01, o aluno tem a opção de selecionar a disciplina. Esse aluno está matriculado no curso de Sistemas de Informação e possui as disciplinas de Desenvolvimento Avançado, Web, OAC e Redes.

Figura 01 - Lista disciplinas do aluno.

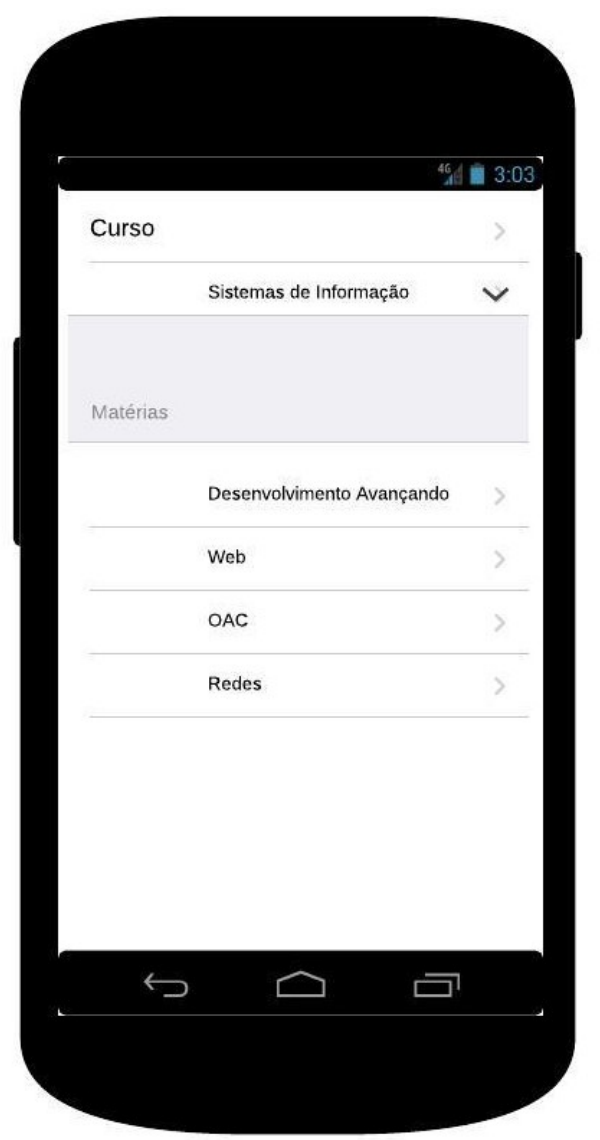

Fonte: PrintScreen Aplicativo, 2018.

Ao selecionar a disciplina o aluno tem acesso aos materiais e aos OAs disponíveis no ambiente. Um exemplo, ao selecionar a disciplina de Desenvolvimento avançado o mesmo tem acesso às mídias digitais disponíveis. Na Figura 02, pode ser observar as mídias disponíveis. 
Figura 02 - Lista de mídias da discicplina.



Fonte: PrintScreen Aplicativo, 2018.

As mídias disponíveis são do tipo: vídeo do YouTube, um bot através do Whatsapp, um game, arquivos em .pdf da disciplina, diretório com todos os arquivos e por fim uma avaliação, para verificar se de fato o aluno está apto para passar para o próximo conteúdo ou não.

Na visão da avaliação que é o principal objeto de estudo deste trabalho, para efetivação do objeto de aprendizagem o aluno visualizará o conteúdo que será avaliado, como pode ser observado na Figura 03.

Figura 03 - Avaliação do conteúdo lonic.



Fonte: PrintScreen Aplicativo, 2018. 
O conteúdo avaliado em questão é o lonic, como falado anteriormente no tópico sobre consolidação da aprendizagem. Não pode se ter sobrecarga cognitiva ao aprender um novo conhecimento, por isso da necessidade de um único tópico ser ministrado de cada vez. Portanto, se o aluno conseguir resolver as questões proposta a respeito do conteúdo de lonic, será liberado automaticamente o próximo tópico para revisão e aprendizagem. Caso negativo, esse aluno receberá mais conteúdos sobre lonic e inclusive o apoio do Tutor/Professor para atuar diretamente nas dúvidas do aluno. $\mathrm{Na}$ Figura 04 pode se observar essa interação do Tutor/Professor com o aplicativo.

Figura 04 - Tela de interação Tutor/Professor.

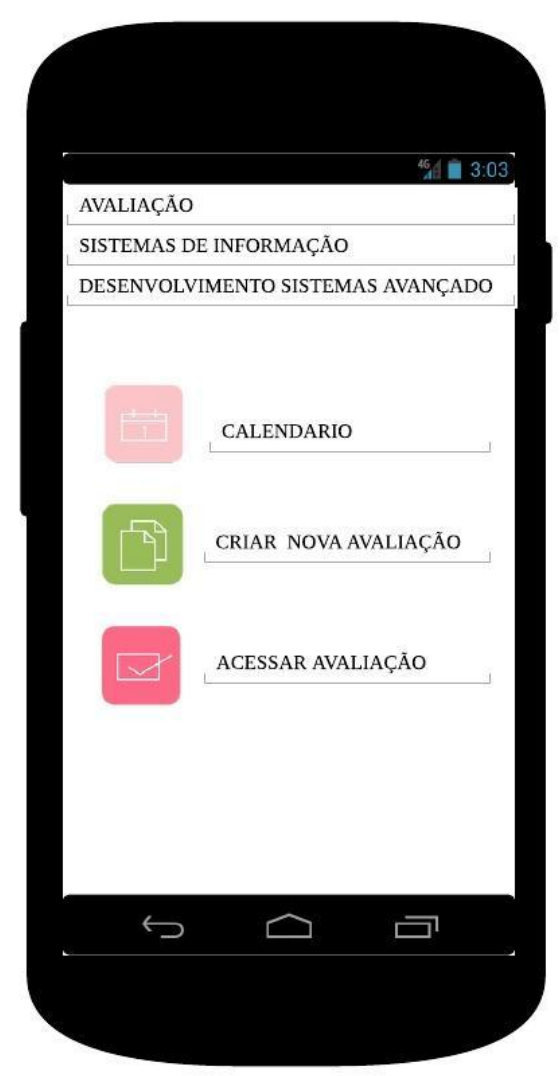

Fonte: PrintScreen Aplicativo, 2018.

\section{CONSIDERAÇÕES FINAIS}

A presente pesquisa buscou validar a efetividade dos objetos de aprendizagem que são disponibilizados para os alunos atualmente. Um objeto de aprendizagem deve ser chamado dessa forma se de fato o mesmo efetivar a aprendizagem.

Dessa forma um objeto de avaliação é inserido a cada objeto de aprendizagem 
ministrado, para averiguar se realmente a aprendizagem foi consolidada. Seguindo os métodos do mapa de conhecimento estruturado, se o aluno não conseguir comprovar por meio da avalição ou uma prática/ação, inserida pelo tutor/professor o mesmo não pode passar para o próximo conteúdo, sendo necessário receber mais conteúdos e ter o apoio dos tutores/professores para sanar suas dúvidas.

Sendo assim esse aplicativo desenvolvido que avalia a efetividade de objetos de aprendizagem, permite quantificar e qualificar a aprendizagem do aluno. Possibilitando a geração de relatórios e registros que identifica de maneira personalizada a dificuldade que cada um possui. Possibilitando uma melhor atuação da instituição quanto a dificuldade de cada estudante.

\section{REFERÊNCIAS}

BEHAR, Patrícia A.; TORREZZAN, Cristina A. W. Parâmetros para a construção de materiais educacionais digitais do ponto de vista do design pedagógico. In: BEHAR, Patrícia A. (Cols.). Modelos Pedagógicos em Educação a Distância. v.1. Porto Alegre: Artmed, 2009. P.33-65.

COWAN, Nelson. (2001). The magical number 4 in short-term memory: a reconsideration of mental storage capacity. Behavioral and Brain Sciences.

DIAS, D. C; COSTA, N. da S.; LIMA, L. V. Projeto e implementação de recursos didáticos multimídia interativos para melhoria do ensino de disciplinas de engenharia de computação. Buenos Aires: COPEC, 2009.

FERREIRA, Daniela Carvalho Monteiro; LIMA, L. V. ; CAMARGO JUNIOR, H. ; SCHIOVATO, N. S. C. . Mapas de Conhecimento Estruturado: proposta de uma nova abordagem metodológica de ensino e aprendizagem. Educere et Educare (versão eletrônica), v. 9, p. 505-514, 2014.

INSTITUTE OF ELECTRICAL AND ELECTRONICS ENGINEERS (IEEE). Draft Standard for Learning Object Metadata. Learning Technology Standards Committee. jul. 2002. Disponível em: Acesso em: 02 abr. 2018.

MOREIRA, M. et al. (2006) "A EaD no Processo de Democratização do Ensino Superior no Brasil", Desafios da Educação a Distância na Formação de Professores, Brasília, Secretaria de Educação a Distância - Ministério da Educação, p. 191-210. 
MILLER, George. The magical number seven, plus or minus two: some limits on our capacity for processing information. Psychological Review, v. 101, n. 02, p. 343-352, 1956.

MIRANDA, R. M. (2004 p.14) "GROA: Universidade Federal do Rio Grande do Sul. Um gerenciador de repositórios de objetos de aprendizagem”. 80p, il. Tese (Mestrado).

NIKOLOPOULOS, G.; SOLOMOU, G.; PIERRAKEAS, C.; KAMEAS, A. Modeling the characteristics of a learning object for use within e-learning applications. BCl'12. Proceedings of the Fifth Balkan Conference in Informatics. p. 112-117., NY, USA: ACM New York, 2012.

SPINELLI, W. Os objetos virtuais de aprendizagem: ação, criação e conhecimento. 2007.

www.lapef.fe.usp.br/rived/textoscomplementares/textolmodulo5.pdf. Acesso em: Acesso em: 07 mar. 2018.

SOSTERIC, M.; Hesemeier, S. (2002). "When is a Learning Object not an Object: A first step toward a theory of learning objects". International Review of Research in Open and Distance Learning. Disponível em: Acesso em: 07 mar. 2018.

SWELLER, J. Evolution of human cognitive architecture. In: ROSS, B. The Psychology of Learning and Motivation. San Diego: Academic Press. 2003, p. 215-266.

TAVARES, Romero. Aprendizagem Significativa. Revista Conceitos, 55-60. 2003. 